\title{
Weak and semi-strong solutions of the Schneider-Tricomi problem in the euclidean plane
}

\section{John M.S. Rassias}

Schneider (Math. Nachr. 60 (1974), 167-180) has established the following result. Consider the mixed type equation

$$
L[u]=k(y) \cdot u_{x x}+u_{y y}+\lambda(x, y) \cdot u=f(x, y)
$$

in $G \subset R^{2}$ which is a simply connected region, bounded for $y>0$ by a piece-wise smooth curve $\Gamma_{0}$ connecting the points $A(0,0)$ and $B(1,0)$, and for $y<0$ by the solutions of $k(y) \cdot(d y)^{2}+(d x)^{2}=0$ which meet at the point $G\left(\frac{7}{2}, y_{c}\right)$, such that $k(y) \gtrless 0$ for $y \geqslant 0$,

(2) $\left\{\begin{array}{r}k(y) \in C^{0}(\bar{G}) \cap c^{1}(\bar{G} \mid\{(x, 0) \mid x \in[0,1]\}) \cap c^{2}\left(G_{2}\right), \\ G_{1}=G \cap\{y>0\}, G_{2}=G \cap\{y<0\}, \lambda=\text { const. }<0, \\ \lambda \in C^{1}(\bar{G}), f \in L^{2}(\bar{G}), \quad u \in C^{0}(\bar{G}) \cap c^{1}(\bar{G}), k^{\prime}(y)>0 \text { in } \\ \bar{G} \cap\{y<0\}, \underset{y \rightarrow 0-}{\lim } \frac{k(y)}{k^{\prime}(y)}=0,\end{array}\right.$

$S(x, y)=F(y)+8 \lambda \cdot\left(k / k^{\prime}\right)^{2}>0$ in $\bar{G} \cap\{y<0\}$, "Schneider's Condition", where $F(y)=1+2\left(k / k^{\prime}\right)^{\prime}$, and such that

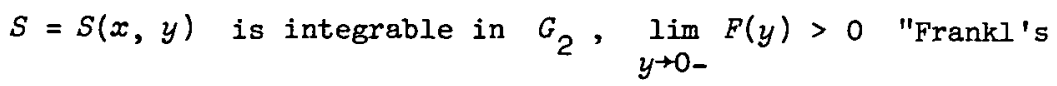
Condition". Then the Tricomi Problem $(T): L[u]=f$ with

Received 19 January 1979. 
$\left.u\right|_{\Gamma_{0} \cup B C}=0$ has a weak solution $u \in L^{2}(\bar{G})$, and the Adjoint Tricomi Problem $\left(\mathrm{T}^{+}\right): L^{+}[w]=L[w]=f$ with $\left.w\right|_{\Gamma_{0} \cup A C}=0$ has at most one semistrong solution.

In this present paper we get the above result of Schneider in a much more generalized way, so that here our uniqueness theorem and existence results include cases where $S(x, y)$ may be negative in $G_{2}$.

\section{Preliminary terminology}

The Sobolev spaces $\tilde{W}^{2,2}(G)$ and $W^{2,2}(G)$ are defined as follows:

$$
w^{2,2}(G)=\left\{u \mid u(x, y) \in L^{2}(G), D^{a} u \in L^{2}(G) \text { for }|a| \leq 2\right\}
$$

with norm $\|\cdot\|_{2}$ and scalar product $(\cdot, \cdot)_{2}$;

$$
\begin{aligned}
& \tilde{W}^{2,2}(G)=\left\{u\left|u(x, y) \in C^{2}(\bar{G}), u\right|_{\Gamma_{0} \cup B C}=0\right\},[1] ; \\
& C_{0}^{\infty}(G) \subseteq \tilde{W}^{2,2}(G) \subseteq C^{2}(\bar{G}) ; \\
& W^{0,2}(G)=L^{2}(G) ; \tilde{W}^{2,2}(G)+=\left\{w\left|w \in C^{2}(\bar{G}), w\right|_{\Gamma_{0} \cup A C}=0\right\} .
\end{aligned}
$$

$W^{2,2}(G$, bd) is the Sobolev space with special boundary values, $w^{2,2}\left(G, \mathrm{~b}^{+} \mathrm{d}\right)=\left\{w \in w^{2,2}(G) \mid(L[u], w)_{0}=\left(w, L^{+}[w]\right)_{0}, \forall u \in w^{2,2}(G, \mathrm{bd})\right\}$

$$
=\left\{w\left|w(x, y) \in C^{2}(\bar{G}), w\right|_{\Gamma_{0} \cup B C}=0\right\} .
$$

LEMMA 1 [1]. For the existence of a semistrong solution of ( $T$ ) (that is $u \in L^{2}(G)$ such that $\left(u, L^{+}[w]\right)_{0}=(f, w)_{0}$, for all

$w \in w^{2,2}\left(G, b^{+} d\right)$ ) it is necessary and sufficient that

$$
\|w\|_{0} \leq C \cdot\left\|L^{+}[w]\right\|_{0},
$$


where $C=$ const. $>0$ for all $w \in w^{2,2}\left(G, \mathrm{~b}^{+} \mathrm{d}\right)$.

LEMMA 2 [1]. For the existence of a semistrong solution of $(T)$ (see [1]) it is necessary and sufficient that

$$
\|u\|_{0} \leq C \cdot\|L[u]\|_{0},\|w\|_{0} \leq C \cdot\left\|L^{+}[w]\right\|_{0},
$$

where $C=$ const. $>0$ for all $u \in W^{2,2}(G, \mathrm{bd})$, and for all $w \in w^{2,2}\left(G, b^{+} d\right)$.

The Schneider-Tricomi problem

We investigate the expression

$$
2(Z[u], L[u])=2 \cdot \iint_{G} Z[u] \cdot L[u] \cdot d x d y,
$$

where

$$
\left\{\begin{array}{l}
Z[u]=a(x, y) \cdot u \text { in } \bar{G}_{1}, \\
\text { and } \\
Z[u]=a(x, y) \cdot\left[u+4 \cdot\left(\sqrt{-k} \cdot e^{B \cdot x} \cdot u_{x}+u\right) \cdot\left(k / k^{\prime}\right)\right] \text { in } \bar{G}_{2},
\end{array}\right.
$$

where

(7)

$$
\left\{\begin{array}{r}
a=a(x, y)=\exp \left(\int_{0}^{y} 4 \lambda \frac{k(t)}{k^{\prime}(t)} \cdot d t\right) \\
\cdot\left\{a_{0}+\int_{0}^{y} \beta_{0} \cdot\left(t-y_{c}\right) \cdot \exp \left[-\int_{0}^{t} 4 \lambda \frac{k(s)}{k^{\prime}(s)} \cdot d s\right] \cdot d t\right\} \\
\quad \text { in } \bar{G}_{2}\left(a_{0}<0, \beta_{0}>0\right), \\
\text { and } \\
a=a(x, y)=a_{0}-\left(\beta_{0} \cdot y_{c}\right) \cdot y \quad \text { in } \vec{G}_{1} .
\end{array}\right.
$$

We apply Schneider's conditions:

(8) $a=a(x, y) \in c^{2}\left(\bar{G}_{1}\right) \cup c^{2}\left(\bar{G}_{2}\right), b=b(x, y)$,

$$
c=c(x, y) \in C^{1}\left(G_{1}\right) \cup C^{\perp}\left(G_{2}\right) \text {, }
$$

$\bar{G}_{1} \cup \bar{G}_{2}=\{(x, 0) \mid x \in[0,1]\}, 2|x y|=\rho \cdot x^{2}+1 / \rho \cdot y^{2} \quad(\rho>0)$, 
(9) $a^{+}-a^{-}=0, b^{+}-b^{-}=0, c^{+}-c^{-} \leq 0,\left(a_{y}^{+}-a_{y}^{-}\right)+\left(c^{-}-c^{+}\right) \cdot \lambda \geq 0$. In $\bar{G}_{i}:$

(10)

$$
\left\{\begin{array}{l}
\tilde{A}=-k(y) \cdot\left(b_{x}-c_{y}\right)+c \cdot k(y)-2 k \cdot a-\rho_{1} \cdot b^{2}=0, \\
\tilde{C}=\left(b_{x}-c_{y}\right)-2 a-\rho_{1} \cdot c^{2}=0, \tilde{A} \tilde{C}-\tilde{B}^{2}=0, \\
\text { with } \\
\tilde{B}=-k(y) \cdot c_{x}-b_{y}-\rho_{1} \cdot b c, \\
\tilde{D}=k(y) \cdot a_{x x}+a_{y y}+2 \lambda a-\lambda\left(b_{x}+c_{y}\right)-\rho_{2} \cdot a^{2} \geq d_{0}>0,
\end{array},\right.
$$

where $\rho_{i}>0 \quad(i=1,2)$;

$$
\begin{gathered}
\left.(b \cdot d y-c \cdot d x)\right|_{\Gamma_{0}} \geq 0,\left.(b \cdot d y+c \cdot d x)\right|_{\Gamma_{I}} \leq 0, \\
{\left.\left[-d(a \cdot \sqrt{-k})+\left(b \cdot \lambda-k \cdot a_{x}\right) \cdot d y+\left(-c \cdot \lambda+a_{y}\right) \cdot d x\right]\right|_{\Gamma_{1}(=A G)} \geq 0,}
\end{gathered}
$$

where

$$
\Gamma_{1}: x=-\int_{0}^{y} \sqrt{-k(t)} \cdot d t
$$

In $G_{1}:$

$$
\tilde{A}=-2 k \cdot a \geq 0, \tilde{C}=-2 \cdot a \geq 0, \tilde{B}=0, \tilde{D}=2 \lambda \cdot a-\rho_{2} \cdot a^{2} \geq 0,
$$

because $\lambda<0$ by hypothesis, $\left.(b \cdot d y-c \cdot d x)\right|_{\Gamma_{0}}=0$. In $G_{2}:$

$\left.(b \cdot d y+c \cdot d x)\right|_{\Gamma_{1}}=\left.\left[b\left(\frac{-1}{\sqrt{-k}}\right)+c\right] \cdot d x\right|_{\Gamma_{1}}=-\left.(-k)^{-\frac{2}{2}} \cdot c \cdot[-k \cdot R(x)] \cdot d x\right|_{\Gamma_{1}}$ $=-\left.c \cdot R(x) d x\right|_{\Gamma_{1}}=0$

Assume

$$
\lim _{y \rightarrow 0_{-}} \frac{k(y)}{k^{\prime}(y)}=0
$$

and choose 


$$
b=c \cdot \sqrt{-k} \cdot e^{\beta \cdot x}, c=\frac{4 a k}{k} \text { in } \bar{G}_{2} \text {, }
$$

where $a=a(x, y)$ is defined by (7), and $\beta$ is a given positive constant such that

$$
R(x)=e^{\beta \cdot x}-1 \geq 0 \text {. }
$$

$\tilde{A} \geq 0$ and $\tilde{B} \geq 0$ if (in $\bar{G}_{2}$ )

$$
\begin{aligned}
R(x, y)=F(y)+8 \lambda \cdot\left(k / k^{\prime}\right)^{2}+2 \beta \cdot & \left((-k)^{3 / 2} / k^{\prime}\right) \cdot e^{\beta \cdot x} \\
& =S(x, y)+2 \beta \cdot\left((-k)^{3 / 2} / k^{\prime}\right) \cdot e^{\beta \cdot x}>0
\end{aligned}
$$

in $\bar{G}_{2}$. On the other hand, $\tilde{A} \tilde{C}-\tilde{B}^{2} \geq 0$ in $\bar{G}_{2}$ if

$$
V(x, y)=A \cdot F^{2}+B \cdot F+C<0
$$

where $A=a^{2} \cdot R^{+}(x)$,

$$
\begin{aligned}
R^{+}(x) & =(-a) \cdot\left[4 \cdot \lambda\left((-k) / k^{\prime}\right)+\beta(\sqrt{-k}) \cdot e^{\beta \cdot x}\right]+\beta_{0} \cdot\left(y-y_{c}\right)>0, \\
B & =4 \cdot\left(R^{+}(x) \cdot a_{y}+\beta \cdot e^{\beta \cdot x} \cdot a \cdot \sqrt{-k}\right) \cdot a \cdot\left(k / k^{\prime}\right), a_{y}=4 \lambda\left(k / k^{\prime}\right) \cdot a+\beta_{0} \cdot\left(y-y_{c}\right), \\
C & =4\left[-(\beta \cdot a)^{2} \cdot e^{2 \beta \cdot x} \cdot k+2 \beta \cdot e^{\beta \cdot x} \cdot a \cdot a_{y}\left(\sqrt{-k}+R^{+}(x) \cdot\left(a_{y}\right)^{2}\right] \cdot\left(k / k^{\prime}\right)^{2} .\right.
\end{aligned}
$$

The Schneider-Tricomi problem, or problem $\left(\mathrm{T}_{\mathrm{S}}\right)$ consists of finding a solution $u \in C^{0}(\bar{G}) \cap C^{\mathcal{1}}(\bar{G})$ assuming prescribed values on $\Gamma_{0} \cup \Gamma_{2}$; that is

$$
\left.u\right|_{\Gamma_{0} \cup \Gamma_{2}}=0
$$

THEOREM. Assume conditions (2), (15), (16), (17), and that $\left.\left[-d(a \sqrt{-k})+b \cdot \lambda \cdot d y+\left(a_{y}-c \cdot \lambda\right) \cdot d x\right]\right|_{\Gamma_{1}} \geq 0, \lambda<0$ in $\bar{G}$, and $S(x, y)=d$ such that $d_{0} \leq d \leq d^{0}$ in $\bar{G}_{2}$; and that $R(x, y)$ is integrable in $\bar{G}_{2}$, $\lim _{y \rightarrow 0-} F(y)>0$. Then the Tricomi problem $\left(\mathrm{T}_{\mathrm{s}}\right)$ has a weak solution $u \in L^{2}(\bar{G})$, and the adjoint Tricomi problem $\left(\mathrm{T}_{\mathrm{s}}^{+}\right)$has at most one semistrong solution $\left(d_{0}=\right.$ const. $<0, d^{0}=$ const. $\left.>0\right)$. 


\section{References}

[1] Ju.M. BerezanskiY, Expansions in eigenfunctions of selfadjoint operators (Translations of Mathematical Monographs, 17. American Mathematical Society, Providence, Rhode Island, 1968).

[2] Ф.Н. Франкль [F. Frankl], "О эадачах C.А. Чаплыгина для смешаных до$n$ сверхзвуновых течении" [On the problems of Chaplygin for mixed sub- and supersonic flows], Buzl. Acad. Sci. URSS Sér. Math. [Izv. Akad. Nauk SSSR] 9 (1945), 121-143.

[3] M.H. Protter, "Uniqueness theorems for the Tricomi problem", J. Rational Mech. Anal. 2 (1953), 107-114.

[4] John Michael Rassias, "Mixed type partial differential equations in $R^{n} "$ (PhD dissertation, University of California, Berkeley, 1977).

[5] Manfred Schneider, "Über schwache und halbstarke Lösungen des TricomiProblems", Math. Nachr. 60 (1974), 167-180.

National Metsovion Polytechnic School,

Chair of Mathematics $A^{\prime}$,

Athens,

Greece. 